\title{
Integration of Cryo-EM into Drug Discovery
}

\section{Sarah Hymowitz}

\section{Genentech, United States}

Structural biology is a critical tool in drug discovery. Structures provide a framework to interpret structure-activity relationships for small molecule projects and generate hypotheses that drive compound design. For antibody or protein-based drug discovery projects, structures can be used to differentiate lead molecules based on their epitope and, more generally, elucidate the molecular mechanism of action of potential clinical candidates. Prior to starting a drug discovery campaign, structures of proteins and protein complexes can lead to a better understanding of biological processes underlying human disease. In addition, the shared need for high quality, well-behaved protein is a key driver of assay development, immunization campaigns and structural studies.

Over the past twenty-five years, crystallography has been the dominant technique for determining structures in support of drug discovery. In recent years, cryo-EM has demonstrated the potential to complement crystallography due to advances in sample preparation, hardware and data processing by delivering similar resolution structures without the need for protein crystallization. Setting up a cryoEM facility to support a variety of discovery, antibody-based and small molecule projects requires investment in microscopes, personnel, informatics, sample preparation and lab space. Having a strategy to integrate the effort into an existing structural biology and drug discovery community is also critical for facilitating uptake of a new technique and maximizing impact on projects. In this talk, I will share our strategy and lessons learned through this process, case studies showing how structures generated using cryo-EM have benefited diverse small molecule, large molecule and discovery projects and potential future directions for cryo-EM and structural biology in drug discovery. 